\title{
ITO layers modified in glow discharge plasma for Nematic Liquid Crystal alignment
}

\author{
Yu. Kolomzarov, P. Oleksenko, A. Rybalochka, V. Sorokin, P. Tytarenko, R. Zelinskyy \\ V. Lashkaryov Institute of Semiconductor Physics, NAS of Ukraine, \\ 41, prospect Nauky,03028 Kyiv, Ukraine; e-mail: zelinski@isp.kiev.ua
}

\begin{abstract}
Influence of ionic and plasma treatment on orienting properties of indium-tinoxide (ITO) films was investigated. The stable tilt angle generation of nematic liquid crystal (NLC) molecules was attended. Dependences of NLC molecules tilt angles on various technological parameters and regimes of ITO film deposition have been shown. Results for oriented film surfaces investigated by atomic-force microscopy showed the viscous-elastic mechanism of NLC molecules alignment by the modified ITO films.
\end{abstract}

Keywords: molecular orientation, reactive cathode sputtering, aligned structures, ITO films.

Manuscript received 09.09.09; accepted for publication 22.10.09; published online 04.12.09.

\section{Introduction}

In recent years, the increased interest to unmechanical methods of alignment, for example, the method of photoalignment $[1,2]$ is observed. The irradiation by the polarized light causes anisotropy of microrelief on substrate surface. This method has no failings like those inherent to the method of mechanical rubbing and allows to effectively control the parameters of photoalignment. Another approach for creation of the homogeneous planar or sloping alignment consists in unmechanical treatment by the sloping beam of particles. Particles can be ions, neutral particles, electrons or their mixture. In relation to the effect of treatment, two types of alignment methods are possible to be distinguished. In the first type, the beam consists of particles with material of alignment that is deposited on the substrate. Deposition can be physical vapor deposition, such as thermal resistive, electron beam etc. $[3,4]$. In the second type of treatment, the beam of particles with the energy 0.3 to $3 \mathrm{keV}$ is used to etch orienting film that was applied before irradiation. According to the reports of authors, the method provides perfect alignment of nematic liquid crystal (NLC) molecules in both organic and inorganic orienting layers [5-8]. We proposed the method of alignment that combines two simultaneous processes into one. On the one hand, it is present the treatment of the surface by ions with energy $0.5-4 \mathrm{keV}$ and passivation of this etched surface by material that was sputtered from a cathode, on the other hand. The results of previous researches [9-13] showed that deposition of silicon oxide or oxides of other materials (germanium, indium etc.) formed on glass substrate, the plane of which is oriented under some angle to the normal of the cathode plane, align structures of these oxides. These aligning structures of oxides at the contact with NLC molecules orient them in the direction that coincides with that of ionic bombardment of substrate in the process of deposition.

In this paper, we report the results of investigation of NLC molecules orientation obtained by ITO films deposited using the developed method. The influence of technological factors both on orienting ability of ITO films and on their electro-physical properties are considered.

\section{Experimental setup}

\subsection{Vacuum deposition setup}

The basic idea of researches consists in creation of ultrafine dielectric nanostructures on substrates by the method of reactive cathode sputtering (RCS) of material of target (diode system). Substrates are located in a plasma column that has a rectangular shape with the width $c$ limited by the inequalities $0<c \leq 8 \mathrm{~mm}$ and the length limited by the sizes of cathode. The plasma column is formed by means of the screen with a niche of the width $b$, which is placed between the cathode and substrate [10]. The distance between the screen and cathode gets out thus to eliminate the glow discharge between the cathode and screen. The screen is placed in the distance less than dark cathode space $L_{\min }$. The cathode target is composed and made of silicon with addition of indium with the surface concentration of the latter between 0 and 100 percents. 


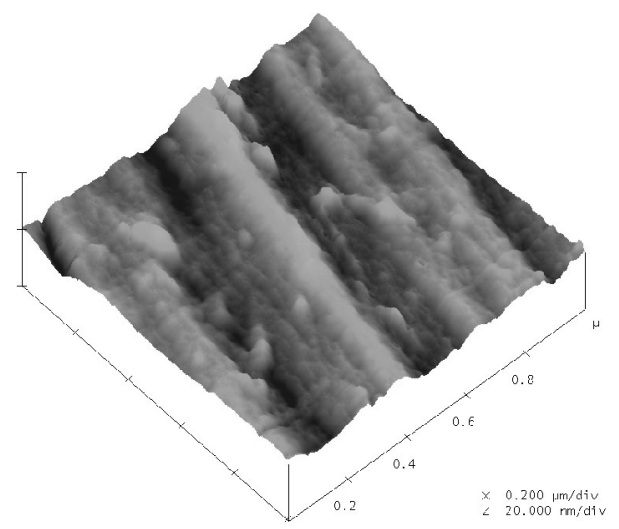

a)

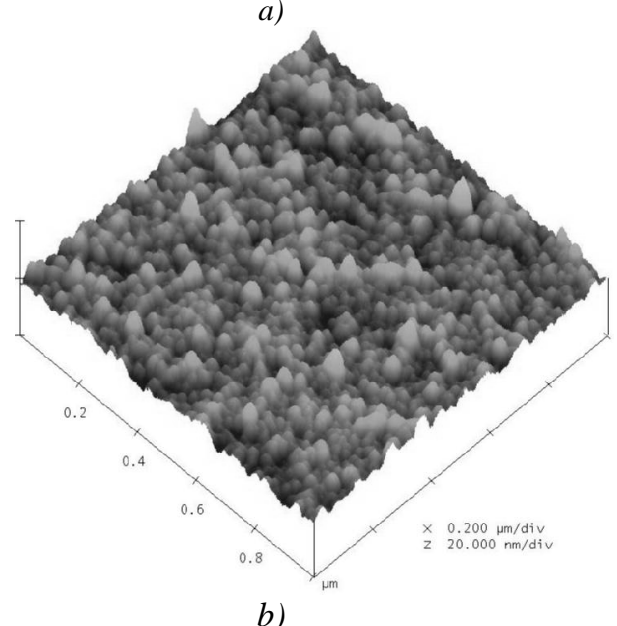

b)

Fig. 1. Topography of aligning layers created by rubbing of cured PI (a) and $\mathrm{SiO}_{\mathrm{x}}$ :In, Sn aligning layer deposited from the target with the concentration of In, Sn alloy $20 \%$ by RCS technology (b).

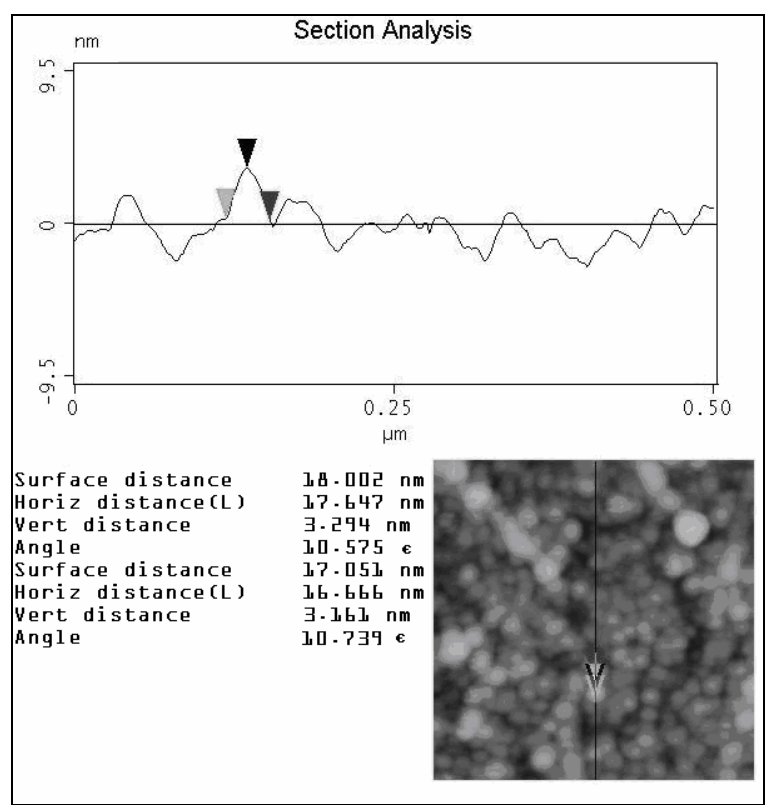

Fig. 2. Section analysis of $\mathrm{SiO}_{\mathrm{x}}: \mathrm{In}, \mathrm{Sn}$ aligning layer deposited from target with concentration of In, Sn alloy $20 \%$ by RCS technology.
Advantages of this cathode target are deposition of orienting layer which is proof to the fumes of organic glue components during substrate sealing and does not require heating the substrates to the temperature of phase transition "NLC - isotropic liquid" during liquid crystal filling. Also, the influence of surface properties on the parameters of orientation is absent. NLC molecules are identically oriented both on the surface of glass and on the surface of ITO electrode. It does not require any preliminary deposition of a dielectric layer from silicon oxide or another material before creation of the orienting layer.

The setup for reactive cathode sputtering of aligning layers is based on standard vacuum setup VUP5. A special water-cooled cathode was mounted at the top part of the bell-jar. As a cathode target, we used silicon wafers that were soldered by indium and tin (5\%) alloy on the cathode plane. Silicon wafers have square shape through holes. The size and arrangement of holes give the possibility to realize three different surface concentrations of indium and tin (5\%) alloy in silicon: $5,10,20,50$ and $100 \%$. The total area of the target was $60 \mathrm{~cm}^{2}$, the square of a sputtered region was $20 \mathrm{~cm}^{2}$. The sputtering of the target was carried out in atmosphere of the argon-oxygen mixture. Substrates were mounted in the special holder enabling to change the slope angle between the normal to plane of the target and substrates within the range from $10^{\circ}$ up to $45^{\circ}$. Special system allows scanning substrates during deposition of aligning layer. The cathode voltage was varied from 1 to $5 \mathrm{kV}$; DC density was varied from 2 to $5 \mathrm{~mA} / \mathrm{cm}^{2}$. Deposition of aligning layers was performed using the pressure of gas mixture within the range from $6 \times 10^{-2}$ to $8 \times 10^{-3}$ Torr. For capillary or vacuum filling, we used LC TL-216.

\subsection{Construction of testing LC cells}

To investigate quality of homogeneous and twist alignment by the polarization microscopy method, we used liquid crystal cells. For preparation of these cells, we used float glass (Glaverbel, thickness $1.1 \mathrm{~mm}$ ) substrates with sizes $20 \times 30 \mathrm{~mm}$. First type samples was spin-coated with polyimide (PI) AD9103 (fabricated in NIIOPIK, Moscow) at $2000 \mathrm{rpm}$ and then annealed at $280^{\circ} \mathrm{C}$ during 1.5 hours. The alignment was created using the standard rubbing process. Alignment in the second type samples was created by the method of $\mathrm{Si}$ target reactive cathode sputtering. After that, on one half of both types of substrates BEN-50 glue (mixture of polyvinylbytiral solution in ethyl alcohol and epoxynovolac block copolymer solution in acetone) was applied, and spacers with diameter $6 \mu \mathrm{m}$ were sprayed onto the other half of substrates.

The substrates of the first and second types were assembled to create homogeneous and twist alignment. After curing of glue (pressure $4 \mathrm{~atm}$, temperature $180^{\circ} \mathrm{C}$, time 1 hour), cells were filled with different types of LC materials. Cells of both types with twist alignment were 
filled with mixture of the LC TL-216 (capillary in air and by vacuum). Cells of both types with homogeneous alignment were filled with LC E7 (capillary in air and by vacuum).

\section{Results and discussion}

Previous investigation showed some imperfections of $\mathrm{SiO}_{\mathrm{x}}$ aligning layers, namely: different aligning properties dependent on previous layer (opened glass, ITO electrode), degradation under the influence of organic glue evaporation and necessity to heat glued substrates during vacuum filling with LC mixture. To exclude these imperfections, cathode targets from silicon and indium were produced. The performed study showed that the orientation quality depends on a great amount of technological parameters during aligning layer deposition (the cathode voltage, discharge current density, gas or gases mixture pressure, oxygen concentration in an operating mixture, angle and time of deposition, etc.).

Our analysis has shown that technological regimes for $\mathrm{SiO}_{\mathrm{x}}$ aligning layers deposition are suitable for deposition of $\mathrm{SiO}_{\mathrm{x}}$ :In aligning layers, which provides the high quality of nematic LC alignment.

The investigation of LC twist and homogeneous structures obtained by substrates with deposited from Si:In, target aligning layers demonstrate the same defectless and perfect on microscopic level alignment. This result is similar to that described in [12]. Alignment is practically independent from previous layers (opened glass, ITO electrode or $\mathrm{SiO}_{2}$ protected layer). It was confirm with optical profilometry investigation of $\mathrm{SiO}_{\mathrm{x}}$ :In, Sn aligning layers deposited on various layers.

To investigate aligning microrelief topography, the AFM method was used. The scanning areas were $1 \times 1 \mu \mathrm{m}^{2}$ and $0.5 \times 0.5 \mu \mathrm{m}^{2}$ for detailed section analysis. The aligning layers were obtained by rubbing of cured

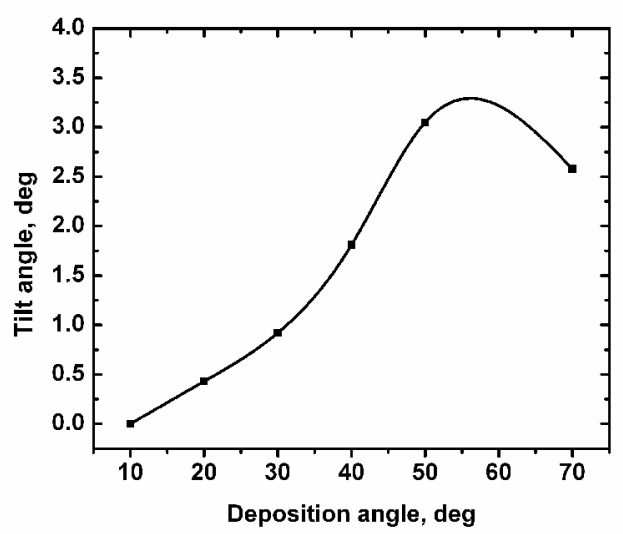

Fig. 3. Dependence of LC molecules tilt angles on the deposition angle. Orientation layer deposition conditions: cathode material - alloy from $95 \% \mathrm{In}$ and $5 \% \mathrm{Sn}, U=$ $2.5 \mathrm{kV}, j=0.7 \mathrm{~mA} \cdot \mathrm{cm}^{-2}$, deposition time $t=10 \mathrm{~min}$, gas mixture $\mathrm{Ar}(80 \%)+\mathrm{O}_{2}(20 \%)$. Capillary filling in air by TL-216 at room temperature.

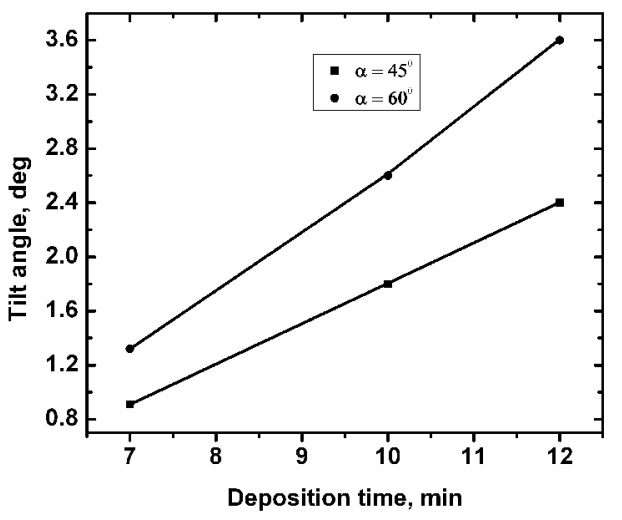

Fig. 4. Dependence of LC molecules tilt angles from deposition times at different deposition angles. Orientation layer deposition conditions: cathode material - alloy from $95 \% \mathrm{In}$ and $5 \% \mathrm{Sn}, U=2.5 \mathrm{kV}, j=0.7 \mathrm{~mA} \cdot \mathrm{cm}^{-2}$, deposition time $t=10 \mathrm{~min}$, gas mixture $\operatorname{Ar}(90 \%)+$ $\mathrm{O}_{2}(10 \%)$. Capillary filling in air by TL-216 at room temperature.

PI and deposited by RCS technology from the target with $20 \%$ of $\mathrm{In}, \mathrm{Sn}$ in $\mathrm{Si}$. Their topographies were shown in Fig. 1a and 1b, respectively. AFM images showed presence of defects in aligning layers created by PI rubbing. This result was confirmed by polarization microscopy investigations of aligned LC structures. In contrast to rubbed $\mathrm{PI}$ the $\mathrm{SiO}_{\mathrm{x}}: \mathrm{In}, \mathrm{Sn}$ aligning layer deposited from target with the concentration of In, Sn alloy $20 \%$ by RCS technology has a defectless microrelief that is able to create the LC aligned structure perfect on a microscopic level.

For full analysis of $\mathrm{SiO}_{\mathrm{x}}: \mathrm{In}, \mathrm{Sn}$ aligning microrelief by AFM, the area with sizes $0.5 \times 0.5 \mu \mathrm{m}$ was scanned in more details. Additionally, the microrelief section parallel to the direction of deposition was analyzed. Topography and section analysis of $\mathrm{SiO}_{\mathrm{x}}: \mathrm{In}, \mathrm{Sn}$ aligning layer deposited from Si target with the concentration of In, Sn alloy $20 \%$ by RCS technology was shown in Fig. 2 . The amplitude of microrelief is several nanometers that correspond to the measurements using the optical profilometry method [12]. The angles of front and rear slopes are approximately 11 degrees. These geometrical parameters explain the uniformity of LC aligned structures and their defectless on a microscopic level.

The tilt angles of NLC molecules in relation to the surface of substrate were measured by the crystal rotation method [13]. Within the range of tilt angles from 0 to $10^{\circ}$, the method provides the error of measurements not worse than $0.1^{\circ}$. This accuracy of measuring the tilt angles is fully acceptable to our study.

Dependences of tilt angles for NLC molecules on different technological parameters are shown in Figs 3 to 6 .

The behavior of tilt angles of NLC molecules depending on the deposition angle and time of saving the liquid-crystal cells filled with LC TL-216 (Figs 3 and 6) coincides with the results of papers [5-8] for the PI 


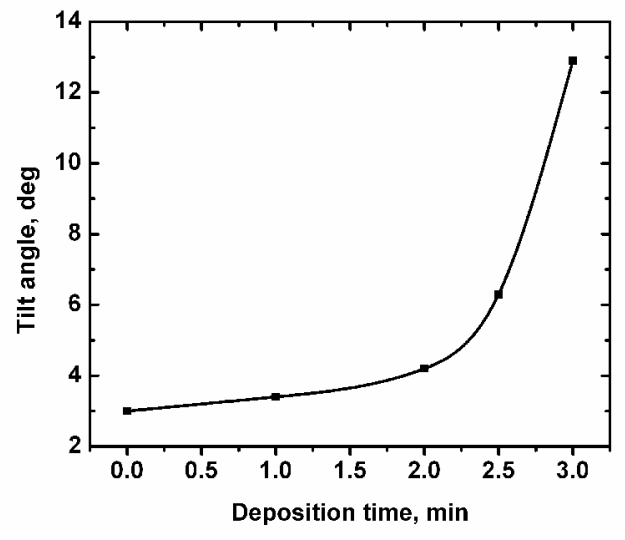

Fig. 5. Dependence of LC molecules tilt angles on the preliminary deposition time. Preliminary layer deposition conditions: cathode material - alloy from $95 \%$ In and $5 \% \mathrm{Sn}$, $U=2.0 \mathrm{kV}, j=0.35 \mathrm{~mA} \cdot \mathrm{cm}^{-2}$, the anode-cathode distance was $50 \mathrm{~mm}$, gas mixture $\mathrm{Ar}(90 \%)+\mathrm{O}_{2}(10 \%)$. Orientation layer deposition conditions: cathode material - alloy from $95 \% \mathrm{In}$ and $5 \% \mathrm{Sn}, U=2.5 \mathrm{kV}, j=0.7 \mathrm{~mA} \cdot \mathrm{cm}^{-2}$, deposition time $t=10 \mathrm{~min}$, gas mixture $\mathrm{Ar}(90 \%)+\mathrm{O}_{2}(10 \%)$. Capillary filling in air by TL-216 at room temperature.

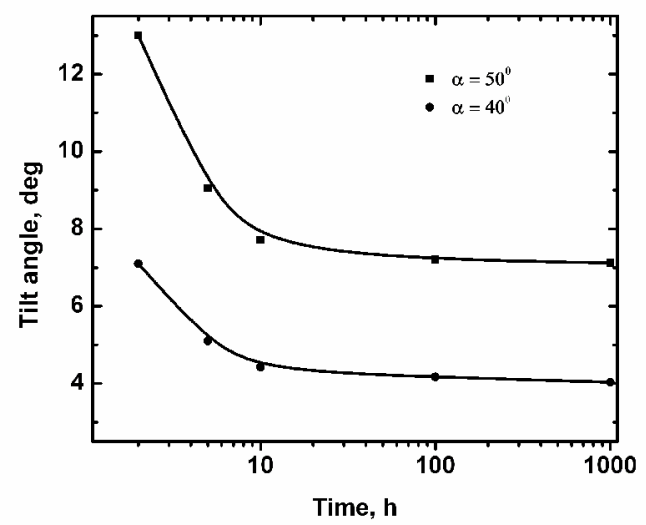

Fig. 6. Dependence of LC molecules tilt angles on the storage time at different deposition angles. Orientation layer deposition conditions: cathode material - alloy from $95 \%$ In and $5 \% \mathrm{Sn}, U=2.5 \mathrm{kV}, j=0.7 \mathrm{~mA} \cdot \mathrm{cm}^{-2}$, deposition time $t=12 \mathrm{~min}$ (6 min deposition, $30 \mathrm{~min}$ cooling in vacuum, 6 min deposition), gas mixture $\operatorname{Ar}(90 \%)+\mathrm{O}_{2}(10 \%)$. Capillary filling in air by TL-216 at room temperature.

orienting films obtained by the method of irradiation of substrate surface by low power ions of argon. In relation to the tilt angles depending on time of deposition of orienting material (or time of treatment of substrate surface), we observed straight proportional dependence of the tilt angle from the deposition time, while the authors of the above mentioned papers observed inverse proportionality between the tilt angle and time of treatments of orienting layer.

It was determined that to obtain a fixed and stable tilt angle of NLC TL-216 molecules on all the operation surface of substrate the preliminary layer by the normal

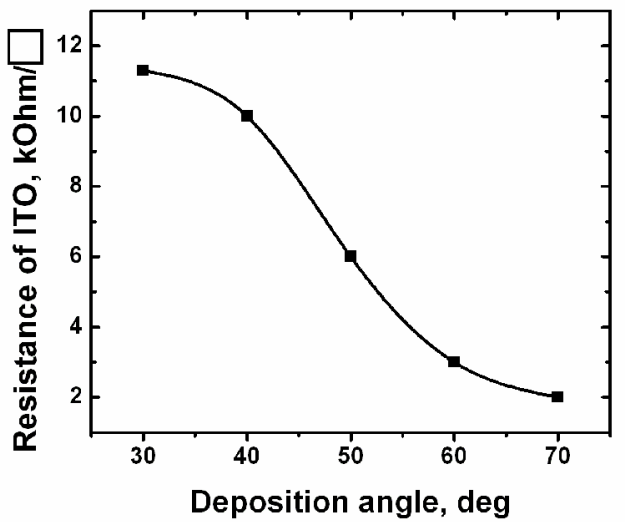

Fig. 7. Dependence of ITO resistance on the deposition angle. Orientation layer deposition conditions: cathode material - alloy from $95 \% \mathrm{In}$ and $5 \% \mathrm{Sn}, U=2.5 \mathrm{kV}, j=$ $0.7 \mathrm{~mA} \cdot \mathrm{cm}^{-2}$, deposition time $t=10 \mathrm{~min}$, gas mixture $\operatorname{Ar}(90 \%)+\mathrm{O}_{2}(10 \%)$. Capillary filling in air by TL-216 at room temperature.

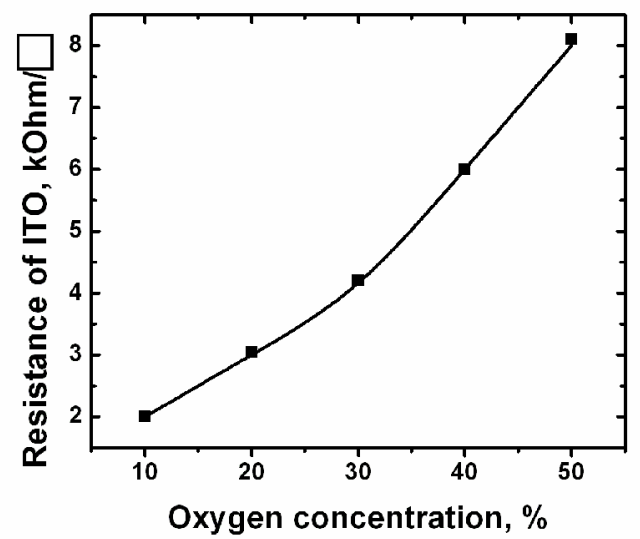

Fig. 8. Dependence of ITO resistance on the oxygen concentration. Orientation layer deposition conditions: cathode material - alloy from $95 \% \mathrm{In}$ and $5 \% \mathrm{Sn}, U=$ $2.5 \mathrm{kV}, j=0.7 \mathrm{~mA} \cdot \mathrm{cm}^{-2}$, deposition time $t=10 \mathrm{~min}$, gas mixture $\operatorname{Ar}(90 \%)+\mathrm{O}_{2}(10 \%)$. Capillary filling in air by TL-216 at room temperature.

to the surface of substrate must be deposited before deposition of orienting layers. Depending on the thickness of this layer, it is possible to get different stable tilt angles of NLC molecules. This feature is demonstrated in Fig. 5.

It was ascertained that deposition of the preliminary layer, on the one hand, is an instrument to increase repeatability of results and, on the other hand, causes undesirable changes in optical and electric properties of ITO layer. Reduction of transmittance of the system ITO-substrate and increase of resistance of 
ITO-electrodes after deposition of preliminary and orienting layers was observed.

Researches of ITO film electric properties showed the substantial increase of their resistance after deposition of preliminary and orienting layers as compared with the initial value of its resistance (Figs 7 and 8 ). It was found that the resistance of ITO films after deposition substantially relies on concentration of oxygen in the operating gas mixture when depositing the orienting layer. As follows from Fig. 8, the change of oxygen concentration from 10 up to $50 \%$ results in the four-time increase of ITO film resistance.

\section{Conclusions}

It is obvious that rubbed PI layer has a lot of imperfections: dust particles in created channels, some channels are not parallel to common rubbing direction. Investigations of the properties of aligning layers deposited by RCS from Si target with the concentration of $\mathrm{In}, \mathrm{Sn}$ alloy $20 \%$ in atmosphere of $\mathrm{Ar}$ and $\mathrm{O}_{2}$ mixture have shown that this method is applicable for creation of NLC molecules alignment in the direction coinciding with ion bombardment and material deposition. The substrate plane should be oriented at the definite angle to the cathode plane normal. Our analysis of LC twist and homogeneous aligned structures by polarization microscopy have demonstrated that $\mathrm{SiO}_{\mathrm{x}}: \mathrm{In}, \mathrm{Sn}$ aligning layer deposited from the target with the concentration of In, Sn alloy $20 \%$ by RCS technology are able to create perfect on microscopic level defectless LC structures. The offered method opens a direct way to create ecologically pure high productive technology of LC displays without using harmful chemical compounds. Investigations by AFM methods have shown that $\mathrm{SiO}_{\mathrm{x}}: \mathrm{In}, \mathrm{Sn}$ aligning layers have an island structure with the height up to $3 \mathrm{~nm}$ in comparison with that in organic continuous layers. This island aligning structure is not an insulator and not produces the voltage drop when external electric field was applied. It is a very important property for characterization of liquid crystal mixtures and gives for researchers the possibility to directly measure the electrical conductivity and dielectric constants of liquid crystals in direct current conditions. Results of investigation of orienting film surfaces by AFM showed the viscous-elastic mechanism of NLC molecules alignment by the modified ITO films. It was ascertained that to obtain the fixed and stable tilt angle of molecules NLC TL-216 on all the working area of substrate before deposition of orienting layers, it is necessary to deposit preliminary a layer by the normal to the surface of substrate. Depending on the thickness of this layer, it is possible to realize various stable tilt angles of NLC molecules. Moreover, using these aligning layers allows creating new types of LC devices.

\section{References}

1. D. Andrienko, D. Fedorenko, O. Uskova, V. Reshetnyak, Yu. Reznikov, S. Slussarenko, D. Voloshchenko, O. Lavrentovich // Ukrainsky fizychny zhurnal 44 (1-2), p. 149-157 (1999), in Ukrainian.

2. E. Ouskova, D. Fedorenko, Yu. Reznikov, S. Shiyanovskii, L. Su, J. West, O. Kuksenok, O. Francescangeli, F. Simoni // Phys. Rev. E 63, 021701 (2001).

3. J. Janning // Appl. Phys. Lett. 21(4), p. 173-174 (1972).

4. W. Urbach, M. Boix, and E. Guyon // Appl. Phys. Lett. 25(9), p. 479-481 (1974).

5. Z.M. Sun, J.M. Engels, I. Dozov, and G. Durand // J. Phys. II France, 4, p. 59-73 (1994).

6. D.J. Kang, J.S. Gwag, J.W. Kang, T.-H. Yoon, J.C. Kim, H. Kim, S.J. Cho // SID-03 Digest, p. 624-627 (2003).

7. J.S. Gwag, K.-H. Park, D.J. Kang, C.G. Jhun, H. Kim, T.-H. Yoon, J.C. Kim // Jpn. J. Appl. Phys. 42, p. L468-L471 (2003).

8. O. Yaroshchuk, R. Kravchuk, A. Dobrovolskyy, S. Pavlov // Proc. Eurodisplay'02, p. 421-424 (2002).

9. V. Sorokin, N. Kuzmin, P. Oleksenko, Y. Kolomzarov, R. Zelinskyy, V. Semenist // Mol. Cryst. Liquid Cryst. 215, p. 137-143 (1992).

10. Yu. Kolomzarov, P. Oleksenko, V. Sorokin, P. Tytarenko, R. Zelinskyy // Semiconductor Physics, Quantum Electronics and Optoelectronics 6(4), p. 528-532 (2003).

11. Yu. Kolomzarov, P. Oleksenko, V. Sorokin, P. Tytarenko, R. Zelinskyy // Proc. SPIE 5565, p. 359-364 (2004).

12. Yu. Kolomzarov, P. Oleksenko, V. Sorokin, P. Tytarenko, R. Zelinskyy // Proc. SPIE 6637, 663709 (2007).

13. Yu. Kolomzarov, P. Oleksenko, V. Sorokin, P. Tytarenko, R. Zelinskyy // Proc. Eurodisplay2007, p. 306-309 (2007).

14. F. Nakano, M. Isogai, M. Sato // Jpn. J. Appl. Phys. 19 (10), p. 2013-2014 (1980). 